\title{
DISCUSSION
}

\section{Monitoring sedimentation of a clay slurry}

\author{
J. BleWETT, W. J. McCARTER, T. M. CHRISP and G. STARRS (2001). Géotechnique 51, No. 8,
} $723-728$

\begin{abstract}
K. Klein, University of Toronto
J. C. Santamarina, Georgia Institute of Technology

The authors measured resistivity at various depths during the sedimentation of kaolinite in different ionic concentration fluids. They attributed a decreasing resistivity (increasing conductivity) with time in the low ionic concentration fluids to decreasing tortuosity. The authors suggested that the initial structure is a card-house, which changes to an aligned structure with time, thereby decreasing the tortuosity.

The purpose of this discussion is to provide complementary information and an alternative hypothesis that may help with the interpretation of these types of studies.
\end{abstract}

\section{THE PORE FLUID}

A dry clay is an electrically neutral system consisting of clay minerals, adsorbed counter-ions and precipitated excess salts. When de-ionised water is added, precipitated salts are hydrated and form the electrolyte. On the other hand, adsorbed hydrated counter-ions remain near the clay surface, together forming the diffuse double layer. If a solution is added, both precipitated salts and adsorbed ions become hydrated. (Note that the solution is presumed not saturated: that is, water molecules in the solution have the ability to hydrate additional ions.) However, there may be ionic exchange between the ions in the solution and the ions in the diffuse double layer. Hence the pore fluid includes hydrated ions that came from the solution, from the precipitated excess salts, and from the exchange with counter-ions. The writers have tested kaolin from Georgia and have found that their natural excess salt is about 0.04 gsalt $/ \mathrm{kg}$ clay.

The conductivity of solutions reflects the availability (i.e. concentration) and mobility of hydrated ions. Therefore the conductivity of the pore fluid, $\sigma_{\mathrm{p}}(\mathrm{S} / \mathrm{m})$ in the clay-solution mixture can be estimated as (assuming low concentrations)

$$
\sigma_{p}=\sum_{i} c_{i} \Lambda_{i}
$$

where $c_{i}\left(\mathrm{~mol} / \mathrm{m}^{3}\right)$ is the ionic concentration and $\Lambda_{i}$ $\left(\mathrm{Sm}^{2} / \mathrm{mol}\right)$ is the molar conductivity of each ionic species present in the pore fluid (values of $\Lambda$ and a more detailed discussion can be found in Santamarina et al., 2001a). Clearly, the conductivity of a solution increases when mixed with an unwashed clay, owing to the presence of excess salts.

\section{THE CLAY-SOLUTION MIXTURE}

Conduction in wet geomaterials is largely electrolytic, taking place within connected pore spaces. That is, when hydrated ions in the pore fluid are subjected to an electric field, they displace so that cations move towards the cathode and anions move towards the anode. This displacement is hindered by tortuosity (as discussed by the authors), particularly at low frequencies. At high frequencies, the effect of tortuosity gradually decays as ion displacement becomes very small.

In addition to bulk fluid conduction in the pore spaces, ions in the counter-ion clouds near particle surfaces contribute 'surface conduction'. This contribution to global conduction develops even when soils are washed and all excess ions are removed so that the pore fluid is de-ionised water. The importance of surface conduction increases with increasing surface-to-volume ratio of mineral surface aligned with the applied field. Hence the contribution of surface conduction to global conduction increases as the specific surface increases, the porosity decreases, and the main axes of particles are aligned with the field. The chronological development of surface conduction models and their limitations can be found in the following sequence of papers: O'Konski (1960), Schwarz (1962), Schurr (1964), Dukhin \& Shilov (1974), Fixman (1980), Lyklema et al. (1983), Grosse (1988), and Kijlstra et al. (1992).

A simple model for the soil conductivity, $\sigma_{\text {soil }}$, can be written to combine pore fluid and surface conduction, by assuming infinitely long, parallel, platy, non-conductive soil particles subjected to an electric field parallel to the length of particles (Klein \& Santamarina, 2002):

$$
\sigma_{\text {soil }}=n \sigma_{\mathrm{p}}+(1-n)\left(\lambda_{\mathrm{ddl}} \frac{G_{\mathrm{s}} \gamma_{\mathrm{w}}}{g}\right) S_{\mathrm{a}}
$$

where $\sigma_{\mathrm{p}}$ is the pore fluid conductivity, $\lambda_{\mathrm{ddl}}$ is the excess surface charge conduction, $n$ is the porosity, $S_{\mathrm{a}}$ is the specific surface, $G_{\mathrm{s}}$ is the specific gravity of the mineral, $\gamma_{\mathrm{w}}=9.8 \mathrm{kN} / \mathrm{m}^{3}$ is the unit weight of water, and $g=9.8 \mathrm{~m} / \mathrm{s}^{2}$ is gravity. Equation (6) shows that the contribution of the pore fluid is restricted by the porosity of the medium, $n$, and that the contribution of surface conduction increases as porosity decreases.

Tortuosity and surface conduction coexist in real soils. However, there is an important distinction. Tortuosity always hinders conduction: that is, the soil conductivity is always lower than the conductivity of the pore fluid. However, surface conduction can render the conductivity of the soil greater than the conductivity of the pore fluid itself. This is the case when the pore fluid conductivity is low and particles have high specific surface (see equation (6)).

Figure 8 shows the variation in soil conductivity with porosity for the three lowest conductivity (highest resistivity) fluids at a height of $285 \mathrm{~mm}$ in the sedimentation column. The porosities are average values determined from the sedimentation volume. Owing to the presence of high specific surface fines that remain in suspension, the conductivities of the pore fluids are expected to lie between the conductivities of the initial solution and the values measured by the authors for the supernatant fluids in the sedimentation column. Also shown in Fig. 8 are data from Klein \& Santamarina (2002) for two different pore fluids with low ionic concentration. The model in equation (6) is fitted to these data (surface conduction $\lambda_{\mathrm{ddl}}=5.38 \times 10^{-10} \mathrm{~S}$ ). In agreement with the discussion above, the conductivity of 


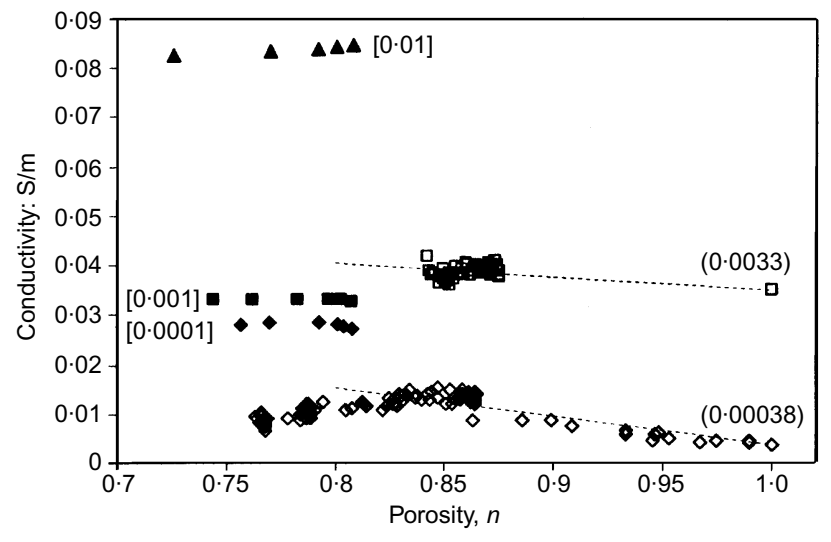

Fig. 8. Conductivity of kaolinite- $\mathrm{NaCl}$ electrolyte slurries as a function of mixture porosity, $n$. The closed symbols are the authors' data, and the concentration of the added solution in $\mathrm{mol} / \mathrm{l}$ is indicated in square brackets. Conductivity values are those measured at a height of $285 \mathrm{~mm}$ in the sedimentation column; porosities are average values determined from the sedimentation volume. The open symbols correspond to data from Klein \& Santamarina (2002); the concentration of the pore fluid in mol/l is indicated in round brackets. These conductivities are measured using an HP-8752A and a coaxial termination probe (frequency $=0 \cdot 1 \mathrm{GHz}$ ). These data are modelled using equation (6) (shown as dashed lines)

mixtures with low pore fluid conductivity decreases with increasing porosity (surface conduction contribution-second term in equation (6)), whereas the mixture conductivity for the high ionic concentration pore fluid increases with increasing porosity (pore fluid contribution-first term in equation (6))

While trends in Fig. 8 and equation (6) point to the relevance of surface conduction, tortuosity also affects the conductivity of soils, as suggested by the authors. The formation of dispersed, flocculated or aggregated particle associations is strongly determined by the $\mathrm{pH}$ and the ionic concentration of the pore fluid. For the pore fluid concentrations used by the authors (all below $\mathrm{c} \approx 0 \cdot 1 \mathrm{M}$ ), the fabric formation changes at the face isoelectric point $($ IEP $\approx 4)$ and at the edge isoelectric point (IEP $\approx 7 \cdot 2$; the fabric map for kaolinite can be found in Santamarina et al., 2001b). Hence it is recommended that both $\mathrm{pH}$ and ionic concentration be documented to facilitate interpreting behaviour that is fabricdependent.

In summary, both tortuosity and surface conduction affect the conductivity of fine-grained soils. Therefore unequivocal physical interpretation requires additional data, such as fabric information.

\section{Authors' reply}

We wish to thank Professor Klein and Professor Santamarina for their alternative interpretation of our results. As a way of continuing this discussion, we wish to offer the following comments on aspects both of the work presented by Professors Klein and Santamarina and of our own.

In general, the intrinsic electrical properties of any non-magnetic material can be fully specified by the frequency-dependent parameters dielectric constant, $\varepsilon_{\mathrm{r}}^{\prime}(\omega)$, and conductivity, $\sigma(\omega)$. These are determined by the polarisation and conduction of bound and free charges within the material. If the material is heterogeneous, $\varepsilon_{\mathrm{r}}^{\prime}(\omega)$ and $\sigma(\omega)$ will be strongly correlated with the properties of the individual components and the way in which they are combined. Such correlation is often manifest in the frequency domain as dispersive behaviour, characterised by frequencies of relaxa- tion, above which dielectric constant falls and conductivity rises (see Fig. 9).

The relative permittivity of the material $\left(\varepsilon_{\mathrm{r}}(\omega)\right)$ is a frequency-dependent complex quantity comprising the dielectric constant, $\varepsilon_{\mathrm{r}}^{\prime}(\omega)$, and the dielectric loss, $\varepsilon_{\mathrm{r}}^{\prime \prime}(\omega)$, related through the expression

$$
\varepsilon_{\mathrm{r}}(\omega)=\varepsilon_{\mathrm{r}}^{\prime}(\omega)-\mathrm{j} \varepsilon_{\mathrm{r}}^{\prime \prime}(\omega)
$$

where $\mathrm{j}$ is the complex operator $\sqrt{ }(-1)$ and $\omega$ is radian frequency. The dielectric constant accounts for energy storage resulting from the polarisation of charges within the material, and the loss factor accounts for the dissipation of energy incurred in the polarisation process, together with any direct transfer of charge through the material. Polarisation could best be described as an oscillation or movement of charges about their zero field equilibrium position. Field, in this context, refers to the applied alternating electrical field. The effects of loss may also be represented as conductivity, $\sigma(\omega)$, which is related to $\varepsilon_{\mathrm{r}}^{\prime \prime}(\omega)$ by the expression

$$
\varepsilon_{\mathrm{r}}^{\prime \prime}(\omega)=\frac{\sigma(\omega)}{\omega \varepsilon_{0}}
$$

where $\varepsilon_{0}$ is the permittivity of free space. The conductivity at a particular frequency will be a combination of both direct conduction processes and dissipative polarisation processes. For example, if the material has a known value of d.c. (or low frequency) ionic conductivity, as is the case for saturated clay, it may be desirable to express the effects of ionic conduction and dissipative effects separately:

$$
\sigma(\omega)=\sigma(0)+\sigma_{\mathrm{d}}(\omega)
$$

where $\sigma_{\mathrm{d}}(\omega)$ is the conductivity due to dielectric relaxation at frequency $\omega$, and $\sigma(0)$ is the low-frequency, ionic conductivity component. The enhancement in conductivity due to increasing frequency is as a result of relaxation of polarisation processes within the system. Interestingly, the two-component format for equation (9) is not dissimilar to equation (6) used by Professors Klein and Santamarina. At high frequencies, the conductivity enhancement due to relaxation processes could dominate the measured conductivity. It is not that the ionic conduction effect disappears or decays; it is simply masked by the dominating effect of dielectric conductivity. In the case of a material such as a clay-water-electrolyte system, conduction, polarisation and relaxation would originate from charges in the pore water and the (diffuse) double layer formed on the surface of the clay particles.

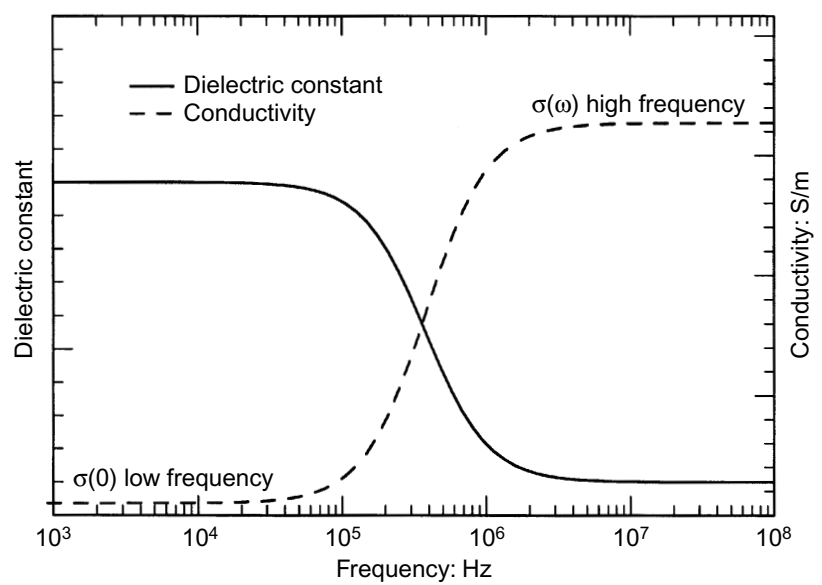

Fig. 9. Idealised dielectric and conductivity dispersion curves showing dispersion due to relaxation of a polarisation process (Note: vertical scales are arbitrary) 
As stated above, the low-frequency conductivity will quantify the ionic conduction effect, whereas the difference between low- and high- frequency conductivity values will represent the enhancement due to relaxation of the polarisation process(es). The 'surface conduction' effect, as invoked by Professors Klein and Santamarina, would represent the contribution due to relaxation of double-layer processes. At very high frequencies (in the $\mathrm{GHz}$ ) relaxation of water molecules will also contribute to an enhancement in conductivity. This being the case, then the frequency at which the electrical measurement is taken becomes important, and the second term in equation (6) would vary with frequency of applied field, as presented in equation (9).

In our experiments we employed the complex impedance formalism to identify the low-frequency conductivity, $\sigma(0)$, which quantifies the bulk ionic conduction effect. The frequency used by Professors Klein and Santamarina in their experiments is several orders of magnitude higher than that used in our tests. At a frequency of $0.1 \mathrm{GHz}$ there could certainly be an influence of dielectric conductivity due to relaxation of double-layer processes (i.e. $\sigma_{\mathrm{d}}(\omega)$ in equation (9) above). It is tacitly assumed that there is negligible contribution from surface process at frequencies in excess of $0.1 \mathrm{GHz}$. We are reasonably confident that the frequencies used in our own work will, in the main, reflect bulk ionic conduction effects, with very little contribution from dielectric relaxation resulting from surface effects.

The difference between the high- and low- frequency conductivity values (i.e. $\sigma(\omega)-\sigma(0)$ ), will give an indication of the enhancement in conductivity due to relaxation (surface) effects. The magnitude of this value will be intimately linked to the bulk ionic conductivity of the system, which, itself, is dictated by the conductivity of the pore fluid, and the porosity and tortuosity of the continuous interstitial path. In general terms, it could be stated that, as the bulk ionic conductivity, $\sigma(0)$, of the system increases, the difference between high- and low- frequency measurements will reduce, implying a reducing influence of surface processes.

Further evidence to suggest that it is the free, mobile ions in the continuous interstitial aqueous phase that provide the principal electrical conduction path at low frequencies can be obtained from consideration of the activation energy for the conduction process. We have undertaken electrical measurements on the variation of conductivity with temperature for the clay system used in our paper. This is presented in an Arrhenius format in Fig. 10 and gives an activation energy, $E_{\mathrm{a}}$, in the region of $17.8 \mathrm{~kJ} / \mathrm{mole}(0.18 \mathrm{eV} /$ ion $)$. Also displayed in this figure is the Arrhenius relationship for an electrolytic solution of similar ionic content and concentration as that within the clay used in this study. The activation energy obtained from these data was $18.0 \mathrm{~kJ} /$ mole.

Equation (5) cited by Professors Klein and Santamarina allows calculation of the ionic conductivity of the pore fluid based on the type and concentration of ions in the pore fluid. In our current paper, we did not undertake this chemical analysis and simply presented the summative effect of all the ions in solution (Table 1). Such analysis would certainly be considered for a more detailed paper on this subject. Finally, we totally agree with the comments regarding the need for additional information (e.g. pH) to interpret

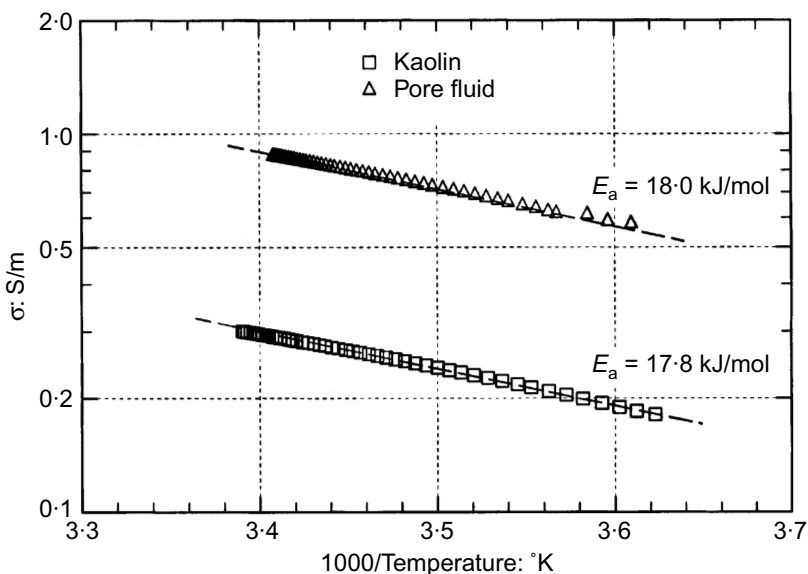

Fig. 10. Variation of conductivity with temperature (plotted in an Arrhenius format)

fabric features of clay soils. Regarding the latter, we have taken (as yet unpublished) environmental scanning electron micrographs on clay microstructure in parallel with electrical measurements.

\section{REFERENCES}

Dukhin, S. S. \& Shilov, V. N. (1974). Dielectric phenomena and the double layer in disperse systems and polvelectrolytes. Toronto: John Wiley \& Sons.

Fixman, M. (1980). Charged macromolecules in external fields. 1. The sphere. J. Chem. Phys. 72, No. 9, 5177-5186.

Grosse, C. (1988). Permittivity of a suspension of charged spherical particles in electrolyte solution. 2. Influence of the surface conductivity and asymmetry of the electrolyte on the low- and high-frequency relaxations. J. Phys. Chem. 92, 3905-3910.

Kijlstra, J., van Leeuwen, H. P. \& Lyklema, J. (1992). Effects of surface conduction on the electrokinetic properties of colloids. $J$. Chem. Soc. Faraday Trans. 88, 3441-3449.

Klein, K. \& Santamarina, J. C. (2002). Electrical conductivity in soils: surface conduction and fabric anisotropy effects. J. Geotech. Geoenviron. Engng (submitted).

Lyklema, J., Dukhin, S. S. \& Shilov, V. N. (1983). The relaxation of the double layer around colloidal particles and the lowfrequency dielectric dispersion. Part I: Theoretical considerations. J. Electroanal. Chem. Interfacial Electrochem. 143, 1-21.

O'Konski, C. T. (1960). Electric properties of macromolecules. V. Theory of ionic polarization in polyelectrolytes. J. Phys. Chem. 64, 605-618.

Santamarina, J. C., Klein, K. \& Fam, M. (2001a). Soils and waves. Toronto: John Wiley \& Sons.

Santamarina, J. C., Klein, K., Palomino, A. \& Guimaraes, M. (2001b). Micro-scale aspects of chemical-mechanical coupling: interparticle forces and fabric. In Chemical behaviour: Chemomechanical coupling from nano-structure to engineering applications (eds C. Di Maio, T. Hueckel and B. Loret). Rotterdam: Balkema.

Schurr, J. M. (1964). On the theory of the dielectric dispersion of spherical colloidal particles in electrolyte solution. J. Phys. Chem. 68, 2407-2413.

Schwarz, G. (1962). A theory of the low-frequency dielectric dispersion of colloidal particles in electrolyte solution. J. Phys. Chem. 66, 2636-2642. 